\title{
Biomimetic fabrication and characterization of an artificial rice leaf surface with anisotropic wetting
}

\author{
YAO Jia, WANG JianNan, YU YanHao, YANG Han* \& XU Ying \\ State Key Laboratory on Integrated Optoelectronics, College of Electronic Science and Engineering, Jilin University, Changchun 130012, China
}

Received January 16, 2012; accepted March 26, 2012

In nature, rice leaves exhibit special anisotropic sliding capabilities. Although researchers have succeeded in fabricating artificial rice leaf structures and realizing the wettability function of the leaf surface, these methods used to date are complex and do not allow the fabrication of surfaces with large area. Herein, we adopted a simple technology — two steps soft transfer to fabricate biomimetic rice leaf. The fabricated surface well reproduced the structures of the rice leaf surface and exhibited a static superhydrophobic property similar to that of the real rice leaf surface. In terms of its dynamic wettability, it clearly exhibited an anisotropic sliding property. Systematic measurements showed that the sliding angles parallel and perpendicular with the vein direction were $25^{\circ}$ and $40^{\circ}$, respectively. The method was simple and reliable, without the need for expensive instruments and complex technologies, which could be used for the rapid fabrication of large-area artificial rice leaf surfaces. We believe that the artificial rice leaf surface fabricated by this method has great potential applications in biomimetic functional surfaces, microfluidics, and so on.

anisotropic wettability, superhydrophobic surface, template transfer, rice leaf surface, functional material, sliding angle

Citation: Yao J, Wang J N, Yu Y H, et al. Biomimetic fabrication and characterization of an artificial rice leaf surface with anisotropic wetting. Chin Sci Bull, 2012, 57: 2631-2634, doi: 10.1007/s11434-012-5220-1

Micro/nanostructures endow plants and animals with special wettability. For example, the micropapillose structures on lotus leaves give them self-cleaning abilities [1]; the bristle microstructures possessed by water striders allow them to stand on water easily [2]; the short wide papillae and nanogrooves that make up the surface of roses have a strong adhesive properties with water droplets [3]. Inspired by nature, researchers have paid great attentions to the investigation of the wetting mechanisms and the fabrication of biological surfaces [4,5]. To date, a number of artificial biosurfaces have been successfully realized by various physical and chemical methods, including template methods, chemical vapor deposition, electrospinning, self-assembly, and laser microfabrication [6-11]. In recent years, rice leaf has attracted much attention because of its unique wettability. It exhibits not only excellent superhydrophobic ability but also special anisotropic sliding properties [12]. Namely,

*Corresponding author (email: yanghan@jlu.edu.cn) water droplets prefer to roll along the veins, and show a slight sliding angle (SA) in this direction. These characteristics helped artificial rice leaf gain importance in a number of fields, including in fluidic direction control, no-loss liquid transportation, and self-cleaning materials [13-18]. At present, many fabrication methods have been used to fabricate biomimetic rice leaf surfaces and realize anisotropic contact angles (CA), including photolithography, wrinkling, electrospinning, and interference lithography [19-22]. For example, Chung et al. [20] produced tunable-aspect-ratio micro-wrinkled surfaces with anisotropic CAs using surface wrinkle technology. Zhang et al. [23] adopted nanoimprint lithography to manufacture a series of two-level hierarchical structures which showed a wide range of anisotropic wetting. Xia et al. [24] reported strong controllable anisotropic wetting grooved surfaces, which were fabricated using two-beam laser interference lithography. Wu et al. [25] realized two-directionally controllable anisotropic wetting using modified four-beam interference lithography. How- 
ever, these studies did not achieve the goal of dynamic superhydrophobicity (small SA); neither did they realize anisotropic sliding property. Recently, Wu et al. [26] made a careful investigation of the rice leaf surface, and proposed a new model to interpret the anisotropic sliding behavior. This model involved "three-level" structures (macro/micro/ nano). By combining photolithography, imprint lithography and nanoparticles coating, they manufactured hierarchical three levels structures. The prepared surfaces possessed controllable sliding anisotropy equivalent to that found in natural rice leaf. Although this approach could realize the structure and function of rice leaf, it was complicated and expensive. In this study, we adopted a simple technology - two steps transcription-to fabricate biomimetic rice leaf. Using the natural rice leaf surface as a template, we adopt two steps soft transfer with elastic polymer materials to directly duplicate the microstructures of the real rice leaf surface. Using scanning electron microscopy (SEM), we carried out a comparative analysis of the microstructures in the natural and biomimetic surfaces. Furthermore, we investigated the static and dynamic wettability of the artificial surface characterized by CA and SA, respectively. We believe that this technology could be widely applied to fabricate artificial rice leaf surfaces.

\section{Materials and methods}

\subsection{Materials}

The primary template used in our experiments was natural green rice leaf, which was picked from the city of Yueyang (Hunan Province, China). The material used in the two times transcription was PDMS (polydimethylsiloxane, Dow Corning, USA). Fluoroalkylsilane (Heptadecafluoro-1,1,2, 2-tetradecyl trimethoxysilane) was purchased from Sikang Company (Fujian Province, China). The anhydrous ethanol was of analytical reagent grade.

\subsection{The fabrication of biomimetic rice leaf}

Figure 1 shows a schematic diagram of the artificial rice leaf fabrication process. First, we intercepted a $5 \mathrm{~cm}$-long natural rice leaf, and carefully washed it by ethanol. After drying in air, the rice leaf was spread and pasted on a glass slide as the primary template. To carry out the first soft imprint lithography, we then poured a PDMS layer with a thickness of approximately $3 \mathrm{~mm}$ onto the primary template. After curing, we peeled the PDMS film off the primary template, and pasted it on another glass slide. This produced the inverse structure template. Finally, the artificial rice leaf was obtained after a second soft transfer following the same process as the first. The detailed processes were as follows: The PDMS prepolymers and curing agent were mixed in a 10:1 weight ratio. During each PDMS soft transfer process, we poured the PDMS on a template, and the PDMS was degassed in vacuum chamber. After baking at $70^{\circ} \mathrm{C}$ for $4 \mathrm{~h}$, the PDMS was solidified and could be peeled off after it had cooled. In the second transcription, the template was composed of PDMS. To facilitate the peeling process, the surface was treated with surface modification before the second transcription. After thermal evaporation at $60^{\circ} \mathrm{C}$, fluoroalkylsilane with low surface energy was grafted on the surface of the structure, which lowered the interfacial adhesion.

\subsection{Sample characterization}

The surface morphologies of the biomimetic rice leaf were characterized using a field emission scanning electron microscope (JSM-7500F, JEOL, Japan). We investigated the surfaces obtained by two steps PDMS soft imprint process. The results were characterized and compared with a natural rice leaf surface. Both the static and dynamic wettability of the surface were studied. To accomplish this, we used a Contact Angle System OCA 20 instrument (DataPhysics, Germany). Static CA measurements were performed using a droplet with a volume of $0.004 \mathrm{~mL}$. We measured the contact angle perpendicular and parallel to the leaf vein, to characterize the CA anisotropy. The dynamic wettability was characterized by measuring the sliding angle (SA). The Contact Angle System was also used for these experiments; a droplet (with a volume of $0.004 \mathrm{~mL}$ ) was placed on the biomimetic rice leaf, and the test bench was tilted until the droplet began to slide. This angle of inclination was taken as the SA.

\section{Results and discussion}

Figure 2 shows a scanning electron microscope (SEM) image of the natural rice leaf. As Figure 2(a) shows, there were macro groove arrays of sub-millimeter scale on the surface. The height and width of the grooves were approximately 200 and $50 \mu \mathrm{m}$, respectively. There were also full papilla structures of micrometer-scale, with heights and widths of approximately 3-5 and 5-8 $\mu \mathrm{m}$, respectively. As shown in the magnified image (Figure 2(b)), abundant nanostructures were clearly present on each papilla. These morphologies constituted the micro/nanostructures of the papilla. These micro/nanostructures offer enough surface roughness to allow the rice leaf to exhibit excellent superhydrophobic characteristics. The macro groove arrays

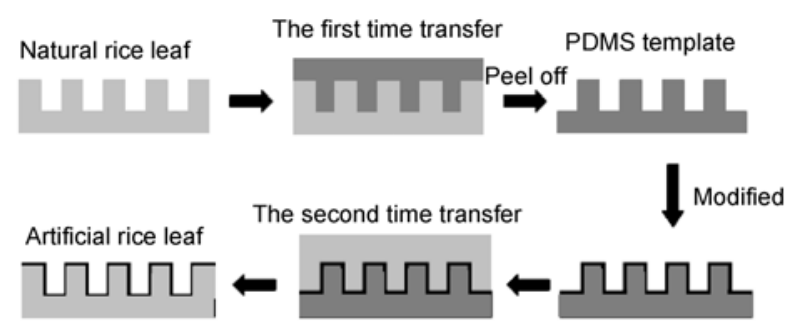

Figure 1 The process used for the fabrication of the biomimetic rice leaf surface. 

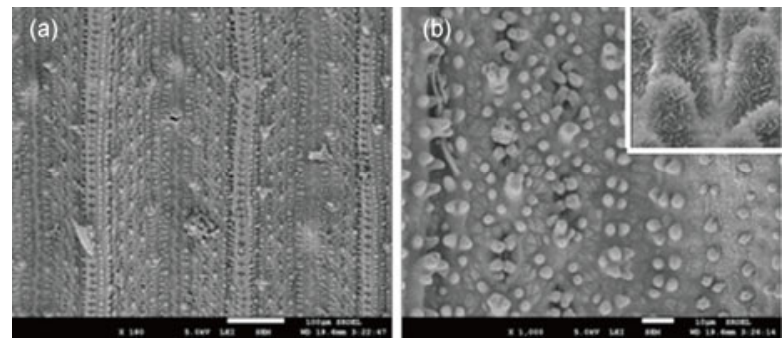

Figure 2 SEM images of a natural rice leaf surface. (a) Macro-groove structure on rice leaf surface; the scale bar represents $100 \mu \mathrm{m}$. The direction along which the groove extends is the vein direction. The SA was smallest in this direction; (b) SEM image of the micro/nano composite structure on the rice leaf surface; the scale bar represents $10 \mu \mathrm{m}$. The inset figure shows a magnified image of the papilla. Nanostructures can be clearly observed. These micro/nano structures mean that the rice leaf has excellent superhydrophobic properties.

extended in one direction (parallel with the vein direction), with the papilla arrays ordered in the same direction. These structural features guide water droplets along the groove, so water droplets exhibit their smallest sliding angle in this direction. Consequently, the rice leaf surface has an anisotropic sliding property.

First, we investigated the microstructures of artificial biomimetic surfaces. Figure 3 shows SEM images of the prepared artificial rice leaf. Figure 3(a) is the inverse rice leaf structure after the first transcription. This structure included macrogroove structures and porous microstructures. Figure 3(b) shows the porous microstructure. These were simply the inverse of the nanostructures on the rice leaf. These results showed that the macro/micro/nano structures of the rice leaf surface were retained after the first soft transfer. Figure 3(c) and (d) shows images of the biomimetic surface after the second soft transfer. Compared with Figure 2, Figure 3(c) shows nearly the same macro/micro structures. Some nanostructures also can be observed in Figure 3(d). The structures of artificial rice leaf surface were well reproduced, similar to that of natural rice leaf.

The wettability of the artificial rice leaf surfaces was measured, and a comparison was made with the natural rice leaf. First, we took static CA measurements. For the natural rice leaf, the CAs perpendicular and parallel to the leaf vein were $151^{\circ} \pm 2^{\circ}$ (Figure 4(a)) and $146^{\circ} \pm 4^{\circ}$ (Figure 4(b)), respectively, demonstrating excellent superhydrophobicity. For the corresponding artificial rice leaf, the static CAs in the two directions were $153^{\circ} \pm 3^{\circ}$ (Figure $4(\mathrm{c})$ ) and $145^{\circ} \pm$ $2^{\circ}$ (Figure 4(d)), respectively. Owing to the excellent texture duplication capabilities and low surface energy property of PDMS, the artificial rice leaf exhibited superhydrophobic properties similar to those of the natural leaf.

As the uniqueness of the rice leaf's wettability is particularly reflected in its anisotropic sliding behavior, the sliding angle (SA) was measured. When a water droplet stays on the artifical rice leaf surface, we tilt the surface till the droplet begins to slide. The angle of inclination at which the droplet begins to slide is defined as the SA. The smaller the $\mathrm{SA}$, the more easily the water droplet slides on a surface,
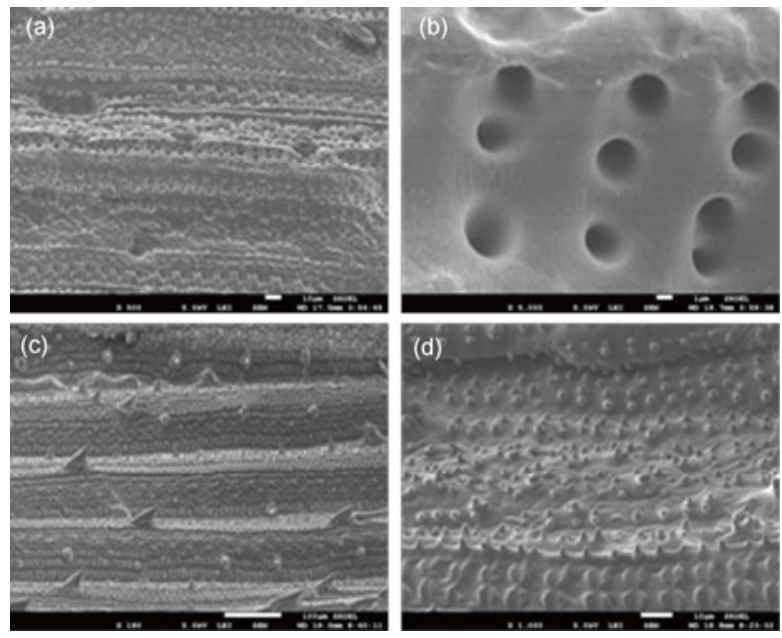

Figure 3 SEM images of the PDMS template and artificial rice leaf. Panels (a) and (b) show SEM images of the PDMS template obtained after the first transfer. The natural leaf macro/micro/nano structures were well preserved on the inverse PDMS surface. (c) and (d) show SEM images of the artificial rice leaf obtained after the second transfer. The macro/micro structures were reproduced very well. The nanostructures were partly reproduced.

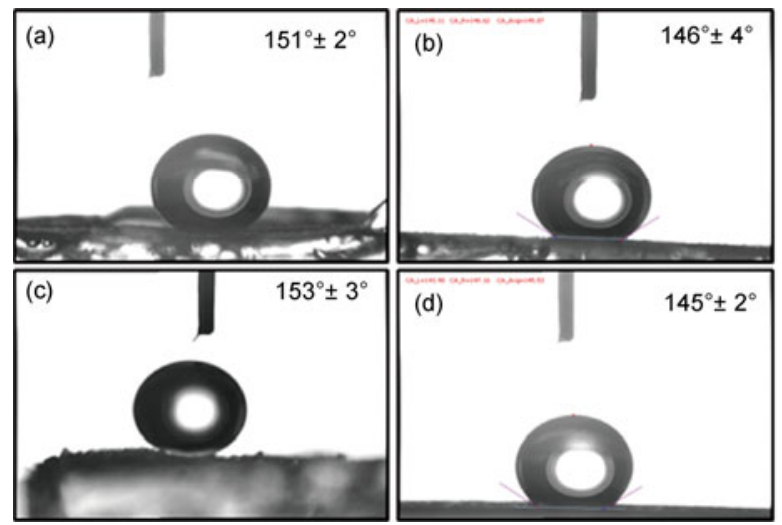

Figure 4 Hydrophobicity tests on the natural and artificial rice leaf. Panels (a) and (b) illustrate the CAs perpendicular and parallel to the natural rice leaf vein direction. Panels (c) and (d) show the CAs perpendicular and parallel to the artificial rice leaf vein direction. There were almost no differences between the two CAs. The fabricated artificial leaf surface well mimicked the static hydrophobicity of a natural surface.

and the better the dynamic wettability of the surface. We took SA measurements in two orthogonal directions. For the natural rice leaf, the SAs along the perpendicular and parallel directions were $9^{\circ}$ and $3^{\circ}$, respectively. For the artificial leaf, the SAs in the two directions were $40^{\circ}$ (Figure 5(a)) and $25^{\circ}$ (Figure 5(b)), respectively. The difference between the CAs in the two directions was small (Figure 4), but the difference in the SAs was large, illustrating the clearly anisotropic sliding properties of the artificial biomimetic surfaces.

\section{Conclusion}

Aiming to replicate the unique wettability of rice leaf, and to address the challenge of the fabrication of an anisotropic sliding surface, we fabricated artificial rice leaf by a simple 
(a)

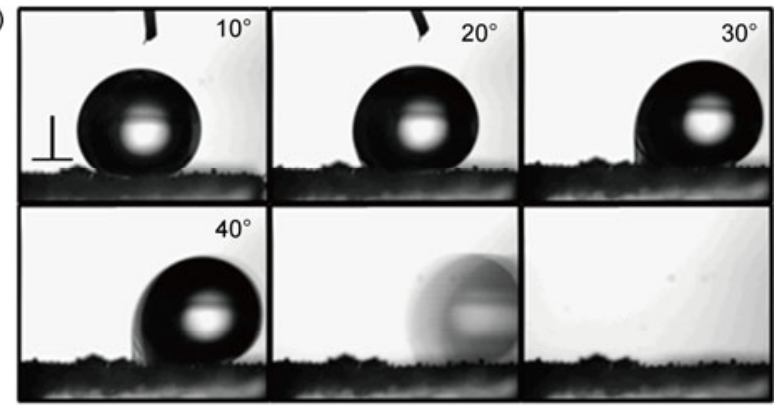

(b)

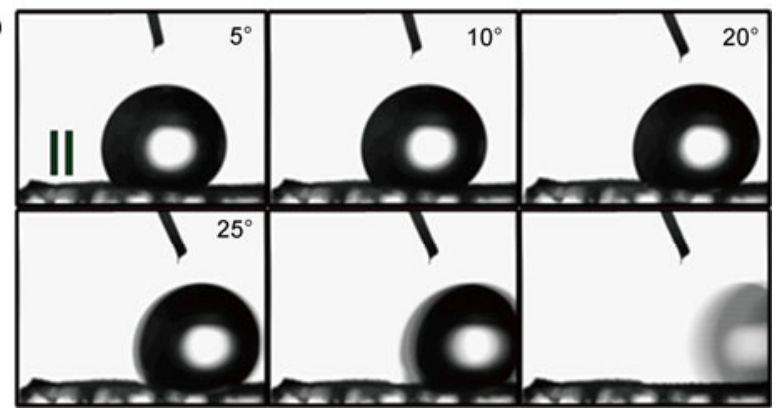

Figure 5 SA test on the artificial rice leaf surface. (a) shows the water droplet's sliding process perpendicular to the vein direction. The water droplet began to slide when the surface was tilted at $40^{\circ}$. (b) shows the water droplet's sliding process parallel with the vein direction; the SA was $25^{\circ}$. The differences between the two directions were large; the surface fabricated by us showed an obviously anisotropic sliding character.

two steps soft imprint method. The achieved surface faithfully reproduced the macroscopic and micro/nanostructures of the natural template, and exhibited directional sliding behavior which is a high contact angle and an anisotropic $\mathrm{SA}$. This method is simple and reliable, without the need for expensive instruments and complex technologies. We believe that the artificial rice leaf surface fabricated by this method could find broader applications in microfluidics, no-loss liquid transportation, and bioinspired systems.

This work was supported by the National Natural Science Foundation of China (60525412, 61077002 and 90923037).

1 Neinhuis C, Barthlott W. Characterization and distribution of waterrepellent, self-cleaning plant surfaces. Ann Bot, 1997, 79: 667-677

2 Gao X F, Jiang L. Water-repellent legs of water striders. Nature, 2004, 432: 36

3 Xi J M, Jiang L. Biomimic superhydrophobic surface with high adhesive forces. Ind Eng Chem Res, 2008, 47: 6354-6357

4 Li M H, He B, Qin H Y, et al. A wet adhesion inspired biomimetic pad with direction dependence and adaptability. Chin Sci Bull, 2011, 56: 1935-1941

5 Hyuneui L, Jung D H, Noh J H. Simple nanofabrication of a superhydrophobic and transparent biomimetic surface. Chin Sci Bull, 2009, 54: 3613-3616

6 Feng L, Song Y L, Zhai J, et al. Creation of a superhydrophobic surface from an amphiphilic polymer. Angew Chem Int Ed, 2003, 115: 824-826

7 Lau K K S, Bico J, Teo K B K, et al. Superhydrophobic carbon nanotube forests. Nano Lett, 2003, 3: 1701-1705

8 Lu X B, Zhou J H, Zhao Y H, et al. Room temperature ionic liquid based polystyrene nanofibers with superhydrophobicity and conductivity produced by electrospinning. Chem Mater, 2008, 20: 3420-3424

9 Srinivasan S, Praveen V, Philip R, et al. Bioinspired superhydrophobic coatings of carbon nanotubes and linear $\pi$ systems based on the "bottom-up" self-assembly approach. Angew Chem Int Ed, 2008, 47: 5750-5754

10 Wang L, Xu B B, Chen Q D, et al. Maskless laser tailoring of conical pillar arrays for antireflective biomimetic surfaces. Opt Lett, 2011, 36: 3305-3307

11 Wu D, Chen Q D, Xia H, et al. A facile approach for artificial biomimetic surfaces with both superhydrophobicity and iridescence. Soft Matter, 2010, 6: 263-267

12 Feng L, Li S H, Li Y S, et al. Super-hydrophobic surfaces: From natural to artificial. Adv Mater, 2002, 14: 1857-1860

13 Haneveld J, Jansen H, Berenschot E, et al. Wet anisotropic etching for fluidic 1D nanochannels. J Micromech Microeng, 2003, 13: 6266

14 Feng R T, Wu X D, Xue Q J. Profile characterization and temperature dependence of droplet control on textured surfaces. Chin Sci Bull, 2011, 56: 1930-1934

15 Gau H, Herminghaus H, Lenz P, et al. Liquid morphologies on structured surfaces. microchannels to microchips. Science, 1999, 283: 4649

16 Wu D, Wu S Z, Chen Q D, et al. Curvature-driven reversible in situ switching between pinned and roll-down superhydrophobic states for water droplet transportation. Adv Mater, 2011, 23: 545-549

17 Fürstner R, Barthlott W. Wetting and self-cleaning properties of artificial superhydrophobic surfaces. Langmuir, 2005, 21: 956-961

18 Fang Y, Sun G, Wang T Q. Hydrophobicity mechanism of nonsmooth pattern on surface of butterfly wing. Chin Sci Bull, 2007, 52: $711-716$

19 Sommers A D, Jacobi A M. Creating micro-scale surface topology to achieve anisotropic wettability on an aluminum surface. J Micromech Microeng, 2006, 16: 1571-1578

20 Chung J Y, Youngblood J P, Stafford C M. Anisotropic wetting on tunable micro-wrinkled surfaces. Soft Matter, 2007, 3: 1163-1169

$21 \mathrm{Wu}$ H, Zhang R, Sun Y, et al. Biomimetic nanofiber patterns with controlled wettability. Soft Matter, 2008, 4: 2429-2433

22 Yang Y L, Hsu C C, Chang T L, et al. Study on wetting properties of periodical nanopatterns by a combinative technique of photolithography and laser interference lithography. Appl Surf Sci, 2010, 256: 3683-3687

23 Zhang F X, Low H Y. Anisotropic wettability on imprinted hierarchical structures. Langmuir, 2007, 23: 7793-7798

24 Xia D Y, Brueck S R J. Strongly anisotropic wetting on one-dimensional nanopatterned surfaces. Nano Lett, 2008, 8: 2819-2824

$25 \mathrm{Wu} \mathrm{S} \mathrm{Z,} \mathrm{Wu} \mathrm{D,} \mathrm{Yao} \mathrm{J,} \mathrm{et} \mathrm{al.} \mathrm{One-step} \mathrm{preparation} \mathrm{of} \mathrm{regular} \mathrm{mi-}$ cropearl arrays for two-direction controllable anisotropic wetting. Langmuir, 2010, 26: 12012-12016

26 Wu D, Wang J N, Wu S Z, et al. Three-level biomimetic rice-leaf surfaces with controllable anisotropic sliding. Adv Funct Mater, 2011, 21: 2927-2932

27 Zheng B, Tice J D, Ismagilov R F. Formation of arrayed droplets by softlithography and two-phase fluid flow, and application in protein crystallization. Adv Mater, 2004, 16: 1365-1368

Open Access This article is distributed under the terms of the Creative Commons Attribution License which permits any use, distribution, and reproduction in any medium, provided the original author(s) and source are credited. 